\title{
The association of depressive symptoms and diabetes distress with glycaemic control and diabetes complications over 2 years in newly diagnosed type 2 diabetes: a prospective cohort study
}

\author{
Khalida Ismail $^{1}$ - Calum D. Moulton ${ }^{1} \cdot$ Kirsty Winkley $^{1} \cdot$ John C. Pickup $^{2}$ • \\ Stephen M. Thomas ${ }^{3} \cdot$ Roy A. Sherwood $^{4} \cdot$ Daniel Stahl $^{5} \cdot$ Stephanie A. Amiel ${ }^{2}$
}

Received: 28 March 2017 / Accepted: 22 May 2017 /Published online: 3 August 2017

(C) The Author(s) 2017. This article is an open access publication

\begin{abstract}
Aims/hypothesis We examined the associations between depressive symptoms and diabetes distress with glycaemic control and diabetes complications over 2 years, after diagnosis of type 2 diabetes.

Methods In a multi-ethnic, primary care cohort $(n=1735)$ of adults, all with recent ( $<6$ months) diagnosis of type 2 diabetes, we measured the associations between depressive symptoms (Patient Health Questionnaire-9 [PHQ-9] score $\geq 10$ ) and diabetes distress (Problem Areas in Diabetes [PAID] score $\geq 40$ ), with change in 2 year $\mathrm{HbA}_{1 \mathrm{c}}$ as the primary outcome and with incident rates of diabetes complications as secondary outcomes. Multivariate models were used to account for potential confounders.

Results Of the 1651 participants $(95.2 \%)$ of the total primary care cohort with available baseline PHQ-9 and PAID scores, mean \pm SD age was $56.2 \pm 11.1$ years, $55.1 \%$ were men and $49.1 \%$ were of non-white ethnicity; $232(14.1 \%)$ and 111
\end{abstract}

Daniel Stahl and Stephanie A. Amiel are joint senior authors.

Khalida Ismail

1 Department of Psychological Medicine, Institute of Psychiatry, Psychology and Neuroscience, King's College London, London SE5 9RJ, UK

2 Division of Diabetes and Nutritional Sciences, Faculty of Life Sciences and Medicine, King's College London, London, UK

3 Department of Diabetes and Endocrinology, Guy's \& St Thomas' NHS Foundation Trust, London, UK

4 Department of Clinical Biochemistry, King's College Hospital NHS Foundation Trust, London, UK

5 Department of Biostatistics, Institute of Psychiatry, Psychology and Neuroscience, King's College London, London, UK khalida.2.ismail@kcl.ac.uk

(6.7\%) had depressive symptoms and diabetes distress, respectively. After adjustment for confounders, depressive symptoms were not associated with worsening $\mathrm{HbA}_{1 \mathrm{c}}$. After adjustment for age, sex, ethnicity, vascular risk factors and diabetes treatments, depressive symptoms were associated with increased risk of incident macrovascular complications (OR 2.78 [95\% CI 1.19, 6.49], $p=0.018$ ) but not microvascular complications. This was attenuated ( $p=0.09$ ) after adjustment for IL-1 receptor antagonist concentration. Diabetes distress was not associated with worsening $\mathrm{HbA}_{1 \mathrm{c}}$ or incident complications.

Conclusions/interpretation In the first 2 years of type 2 diabetes, the effect of depressive symptoms and diabetes distress on glycaemic control is minimal. There was, however, an association between depressive symptoms and incidence of macrovascular complications. Elevated innate inflammation may be common to both depression and macrovascular diabetes complications, but these findings require replication.

Keywords Depressive symptoms · Diabetes distress · Glycaemic control $\cdot$ Inflammation · Macrovascular complications $\cdot$ Microvascular complications $\cdot$ Type 2 diabetes

$\begin{array}{ll}\text { Abbreviations } \\ \text { CABG } & \text { Coronary artery bypass graft } \\ \text { GP } & \text { General practitioner } \\ \text { IL-1RA } & \text { IL-1 receptor antagonist } \\ \text { IMD } & \text { Index of multiple deprivation } \\ \text { IQR } & \text { Interquartile range } \\ \text { LSOA } & \text { Lower Super Output Area } \\ \text { MI } & \text { Myocardial infarction } \\ \text { PAID } & \text { Problem Areas in Diabetes } \\ \text { PHQ-9 } & \text { Patient Health Questionnaire-9 } \\ \text { SOUL-D } & \text { South London Diabetes }\end{array}$




\section{Introduction}

Depressive symptoms are associated with worse outcomes in the natural history of type 2 diabetes mellitus and are associated with an approximately $60 \%$ increased risk of incident disease; conversely, type 2 diabetes increases the risk of incident depressive symptoms by approximately $20 \%[1,2]$. In established type 2 diabetes, depressive symptoms are associated with a 1.5- to threefold increased risk of diabetes complications and premature mortality [3-5]. The reasons for the adverse effects of depressive symptoms in type 2 diabetes are incompletely understood. A psychological explanation is frequently cited for the relationship, namely that depressive symptoms such as low mood and anhedonia (loss of pleasure in everyday activities) lead to neglect of diabetes selfmanagement and consequent worse glycaemic control [6, 7]. However, pooled data from cross-sectional studies of depressive symptoms and glycaemic control have found only a small effect size $(d=0.17)$ [8]. From the few prospective studies published, the evidence for an association between depressive symptoms and glycaemic control is also weak [9-13]. While treatments for depressive symptoms usually improve depressive symptoms [14, 15], they do not always improve glycaemic control, unless integrated with additional support in diabetes management [16]. This suggests that other mechanisms may also underlie the association between depressive symptoms and type 2 diabetes.

An alternative psychological mechanism is diabetes distress. Diabetes distress is distinguished from depression by referring to the burdens, worries and fears specific to people living with diabetes. Measures of diabetes distress, such as the Problem Areas in Diabetes (PAID) scale [17], show moderate-to-strong correlations with self-report measures of depressive symptoms. However, a large part of the variance in such scales is not explained by depressive symptoms [18]. For example, a longitudinal study of 1567 individuals with diabetes found that 55\% of those with diabetes distress did not have likely depression [19], while a factor analysis study reported that depression and distress symptoms could be segregated into two independent factors [20]. Clinically, diabetes distress is significant because it may be a greater psychological barrier to self-management, and therefore to optimising glycaemic control, than depressive symptoms $[21,22]$ and it is potentially modifiable using psychological therapy [23]. To date, we are aware of only one observational study that has compared depressive symptoms and diabetes distress prospectively in type 2 diabetes, reporting that diabetes distress had a greater effect on worsening glycaemic control than on depressive symptoms [9]. We are not aware of a longitudinal study of the independent effect of diabetes distress on increased risk for diabetes complications.

The prevalence of depressive symptoms and diabetes distress are known to increase with diabetes duration [24, 25], an important confounder that limits the interpretation of previous studies of the effects of depression and diabetes distress on self-management and glycaemic control [11, 26]. Studying these potential effects at the time of diagnosis of type 2 diabetes is a methodologically robust approach, as there is minimal confounding by long duration of type 2 diabetes and it is an important window of opportunity for interventions.

We therefore conducted a multi-ethnic cohort study in primary care to test and compare the associations of depressive symptoms and diabetes distress with change in glycaemic control as the primary outcome, as well as incidence of macro- and microvascular complications as the secondary outcomes, in people with newly diagnosed type 2 diabetes.

\section{Methods}

\section{Design}

The South London Diabetes (SOUL-D) study is a populationbased, primary care prospective cohort of people with newly diagnosed type 2 diabetes recruited within 6 months of diagnosis and followed up after 1 and 2 years. Ethical approval was granted by the King's College Hospital Research Ethics Committee (reference 08/H0808/1) and by Lambeth, Southwark and Lewisham Primary Care Trusts (reference RDLSLB 410). All participants gave written informed consent, including for allowing access to their medical records.

\section{Setting}

The study was set in the inner-city boroughs of Lambeth, Southwark and Lewisham in South London, UK, which collectively have approximately 0.75 million residents from diverse socioeconomic and ethnic backgrounds [27]. All general practitioner (GP) surgeries $(n=138)$ in these boroughs were invited to participate. Local protocols for diagnosis of type 2 diabetes followed WHO criteria [28].

\section{Study population and case definition}

People with a recent ( $<6$ months) diagnosis of type 2 diabetes, aged 18-75 years at diagnosis, were identified from the mandatory electronic diabetes registers of participating surgeries and invited to participate. Exclusion criteria, derived from the medical records, were: dementia, terminal illness, temporary residence and residence outside the catchment area, other types of diabetes and severe end-stage diabetes complications defined as registered blind, receiving dialysis or previous above-knee amputation. Recruitment was conducted between May 2008 and April 2011 and follow-up ended August 2014; further details of the sampling methodology have been described in detail elsewhere [27]. The standardised data collection schedule at baseline consisted of a clinical examination, 
self-report questionnaires, venepuncture, urine analysis and data extracted from routine medical records, all repeated at 1 and 2 years $( \pm 3$ months).

\section{Main outcomes}

The primary outcome was $\mathrm{HbA}_{1 \mathrm{c}}$ after 2 years, measured by affinity chromatography (Primus Ultra2; Primus Diagnostics, Kansas City, KS, USA) and reported in both DCCT-derived (\%) and IFCC (International Federation of Clinical Chemists)recommended units $(\mathrm{mmol} / \mathrm{mol})$. $\mathrm{HbA}_{1 \mathrm{c}}$ was also measured at 1 year follow-up. If at follow-up there were missing $\mathrm{HbA}_{1 \mathrm{c}}$ data (participant uncontactable or did not want to attend), we used $\mathrm{HbA}_{1 \mathrm{c}}$ from the medical records if it had been collected 3 months before or after the scheduled date. Secondary outcomes were incidence of macrovascular complications (any myocardial infarction [MI], coronary artery bypass graft [CABG], stroke or carotid/limb revascularisation or amputation, derived from the medical records) and microvascular complications (neuropathy, assessed clinically at each study visit by neurothesiometry vibration perception threshold $\geq 25 \mathrm{~V}$; nephropathy, defined as albumin-creatinine ratio $\geq 3 \mathrm{mg} / \mathrm{mmol}$, taken from medical records; or retinopathy, assessed using medical record retinal eye screen data, performed annually on all residents with diabetes using digital two-field photography and coded by trained graders as present or absent using the English Retinopathy Minimum Grading System) during follow-up.

\section{Main explanatory variables}

Depressive symptoms were measured by the Patient Health Questionnaire-9 (PHQ-9), a nine-item self-report measure based on the Diagnostic and Statistical Manual-IV diagnostic criteria for major depressive symptoms, with scores ranging from 0 to 27 . We used a cut-off score of $\geq 10$ for identifying depressive symptoms, as this has been found to have $88 \%$ sensitivity and specificity for major depression compared with clinical interview [29] and was validated in the SOUL-D cohort at baseline [30]. Diabetes distress was measured using the PAID scale [17]. The PAID is comparable across cultures, has high internal and test-retest reliability and is more suitable for both binary and continuous analysis than other measures of diabetes distress [31-33]. We used a score of 40 or higher to define diabetes distress, as this has been found to be around 1 SD above the mean across different studies and has discriminative validity $[17,31]$. To account for the possible effect of 'subthreshold' symptoms of depression and diabetes distress, we also used both scales as continuous measures.

For participants with $<20 \%$ missing data for either PHQ-9 or PAID, we used case mean substitution to impute missing values. Briefly, case mean substitution imputes missing values based on the mean scores that are present for that participant [34]. For any participant, this strategy assumes that the score on any missing data point is closely related to the scores on the remaining data points. A major advantage of this technique is that it uses data provided by a case to estimate its own missing data, rather than by using data provided by other cases. Case means substitution has been found to be robust in handling item-level missingness when $20 \%$ of data are missing, whether in random or systematic patterns [35].

\section{Sample size calculation}

We estimated that the minimum clinically significant difference in change scores in $\mathrm{HbA}_{1 \mathrm{c}}$ in the depressed group should be $0.5 \%(5.5 \mathrm{mmol} / \mathrm{mol})$ greater than in the non-depressed group, with a prevalence ratio of depressive symptoms to the control group of 1:8 [3]. Using nQuery Advisor, common SD of $1.77, \alpha=0.05,90 \%$ power and $30 \%$ attrition, the total sample required was 1738 people.

\section{Statistical analyses}

Data were analysed using SPSS 22.0 (IBM Corp. Released 2014; IBM SPSS Statistics, Armonk, NY, USA). To assess for potential participation bias, we compared participating and non-participating GP surgeries for list size and index of multiple deprivation (IMD) score, which is an aggregate measure of deprivation across seven domains [36]. The full unit postal code was obtained for each GP surgery and linked with Lower Super Output Area (LSOA), before being assigned an IMD rank. From a total of 32,482 LSOAs in England (UK), a rank of 1 is the most deprived. The baseline characteristics of the sample itself were then stratified by depressive symptoms and diabetes distress status and summarised as mean (SD) or median (interquartile range [IQR]) for normally distributed or skewed data, respectively, or as a count (\%) for categorical variables. We calculated the Pearson's coefficient $(r)$ between depressive symptoms and diabetes distress status at baseline.

For the prospective analysis, we first compared the characteristics of participants who had 2 year $\mathrm{HbA}_{1 \mathrm{c}}$ measured with those of participants lost to follow-up. We next compared the proportion of people with depression and diabetes distress at 2 years, stratified by presence or absence of depressive symptoms and diabetes distress at baseline. We then compared 1 and 2 year $\mathrm{HbA}_{1 \mathrm{c}}$ and number of incident complications stratified by presence or absence of depressive symptoms and diabetes distress at baseline. For continuous variables, we used Student's $t$ test for normally distributed data and Mann-Whitney $U$ test for skewed data, and for categorical variables we used $\chi^{2}$ tests. Our main hypotheses were assessed using multivariable linear regression for $\mathrm{HbA}_{1 \mathrm{c}}$ as outcome and multivariable logistic regression for incident complications as outcome. Finally, we repeated the analyses using continuous PHQ-9 and PAID scores, which were natural $\log$-transformed for multivariate analyses. 
Covariates For all multivariate models, we added age, sex and non-white ethnicity as confounders, as these were associated with depression, glycaemic control and complications in the baseline SOUL-D cohort [25], as well as prescription of hypoglycaemic medications and insulin during follow-up. For the outcome 2 year $\mathrm{HbA}_{1 \mathrm{c}}$, we also controlled for baseline $\mathrm{HbA}_{1 \mathrm{c}}$ to measure worsening of glycaemic control over time. For complications, we added the following vascular risk factors to the model: baseline smoking status; baseline systolic blood pressure (mmHg); baseline fasting serum total cholesterol (measured using Siemens Advia 2400 Analyzer, detection limit $0.01 \mathrm{mmol} / \mathrm{l})$; baseline $\mathrm{HbA}_{1 \mathrm{c}}$ and baseline $\mathrm{BMI}(\mathrm{kg} /$ $\mathrm{m}^{2}$ ). Finally, we added baseline serum concentration of IL-1 receptor antagonist (IL-1RA) to the model as a confounder, as this was the inflammatory cytokine that correlated most strongly with depressive symptoms in a previous study of the cohort [37]. As described in detail previously [37], serum IL-1RA was measured after an overnight fast and analysed using the Randox Evidence Investigator.

\section{Results}

Ninety-six out of 138 (69.6\%) GP surgeries agreed to participate. Participating practices had larger list sizes than those not participating $(10,073 \pm 4962$ vs $5822 \pm 3376, p<0.001)$, but showed no difference in deprivation (IMD rank $7750 \pm 4562$ vs $8254 \pm 4489 p=0.61$ ). From their diabetes registers, a target population of 3008 individuals with newly diagnosed type 2 diabetes were identified during recruitment. Of these, 2406 were potentially eligible and invited to participate and 1735 people consented (Fig. 1).

Of the 1651 people with complete PHQ-9 and PAID assessment at baseline, mean $( \pm \mathrm{SD})$ age was $56.2 \pm 11.1$ years, $55.1 \%$ were men, $49.1 \%$ were of non-white ethnicity and mean $\mathrm{HbA}_{1 \mathrm{c}}$ was $6.99 \pm 1.42 \%(52.9 \pm 10.7 \mathrm{mmol} / \mathrm{mol})$. Of these, $232(14.1 \%)$ and $111(6.7 \%)$ participants had depressive symptoms and diabetes distress, respectively, at baseline. Of those with diabetes distress at baseline, 59 (53.2\%) had comorbid depressive symptoms, while 59 (25.4\%) of those with depressive symptoms had comorbid diabetes distress. The resulting correlation between diabetes distress status and depressive symptoms status was $r=0.30(p<0.001)$.

\section{Baseline comparisons}

At baseline, participants with depressive symptoms were younger, more likely to be women, had higher BMI, were more likely to smoke and had higher baseline IL-1RA concentration. Participants with diabetes distress were younger, more likely to be women and of non-white ethnicity and had lower systolic BP and higher IL-1RA concentration. There were no significant differences in glycaemic control, total cholesterol, diastolic BP or prescription of oral hypoglycaemic medication when participants were stratified by depressive symptoms or diabetes distress (Table 1).

\section{Comparison of dropouts with non-dropouts at 2 years}

At 2 years, those who had $\mathrm{HbA}_{1 \mathrm{c}}$ measured $(n=1234)$ vs those who did not $(n=417)$ were older $(56.8 \pm 10.9$ years vs $54.5 \pm 11.5$ years, $p<0.001)$, had lower baseline $\mathrm{HbA}_{1 \mathrm{c}}$ $(6.91 \pm 1.36 \%[52.0 \pm 10.2 \mathrm{mmol} / \mathrm{mol}])$ vs $7.23 \pm 1.56 \%$ $[55.5 \pm 12.0 \mathrm{mmol} / \mathrm{mol}], p<0.001)$ and were more often of white ethnicity $(53.6 \%$ vs $42.9 \%, p<0.001)$. There were no differences in sex $(p=0.52)$, baseline depressive symptoms status $(p=0.064)$, diabetes distress status $(p=0.37)$, smoking status $(p=0.92)$, prescription of hypoglycaemic medication $(p=0.19)$, serum cholesterol $(p=0.67)$, BMI $(p=0.94)$, systolic BP $(p=0.96)$ or diastolic BP $(p=0.71)$ (data not shown).

\section{Course of depressive symptoms and diabetes distress}

Fifty-four per cent of people with depressive symptoms at baseline still had depressive symptoms 2 years later, while the 2 year prevalence of depressive symptoms (13.8\%) was similar to that seen at baseline. Conversely, only $17.1 \%$ of people with diabetes distress at baseline still had diabetes distress 2 years later, while the overall prevalence of diabetes distress fell to $2.4 \%$ after 2 years.

\section{$\mathrm{HbA}_{1 \mathrm{c}}$}

In univariate analysis, depressive symptoms at baseline were not associated with elevated $\mathrm{HbA}_{1 \mathrm{c}}$ after 1 year. Conversely, depressive symptoms at baseline were associated with elevated $\mathrm{HbA}_{1 \mathrm{c}}$ after 2 years (Table 2) but this was attenuated after adjustment for baseline $\mathrm{HbA}_{1 \mathrm{c}}$, age, sex, ethnicity and diabetes treatments (Table 3). Baseline diabetes distress was not associated with elevated 1 year or 2 year $\mathrm{HbA}_{1 \mathrm{c}}$ in univariate or adjusted models (Table 2 and Table 3 ). Continuous depressive symptoms and diabetes distress were both associated with elevated 2 year $\mathrm{HbA}_{1 \mathrm{c}}$ in univariate analysis. Again, however, differences were attenuated after adjusting for confounders (Table 3).

\section{Complications}

In univariate analysis, there was a positive association between baseline depressive symptoms and incident macrovascular complications. This was largely explained by incident MI, although numbers were low (Table 2). Notably, this association remained significant (OR 2.78) even after adjustment for age, sex, ethnicity, vascular risk factors and diabetes treatments, but was attenuated after adjustment for baseline IL-1RA 
Fig. 1 Flow diagram of recruitment and attrition over 2 years in the South London Diabetes Cohort

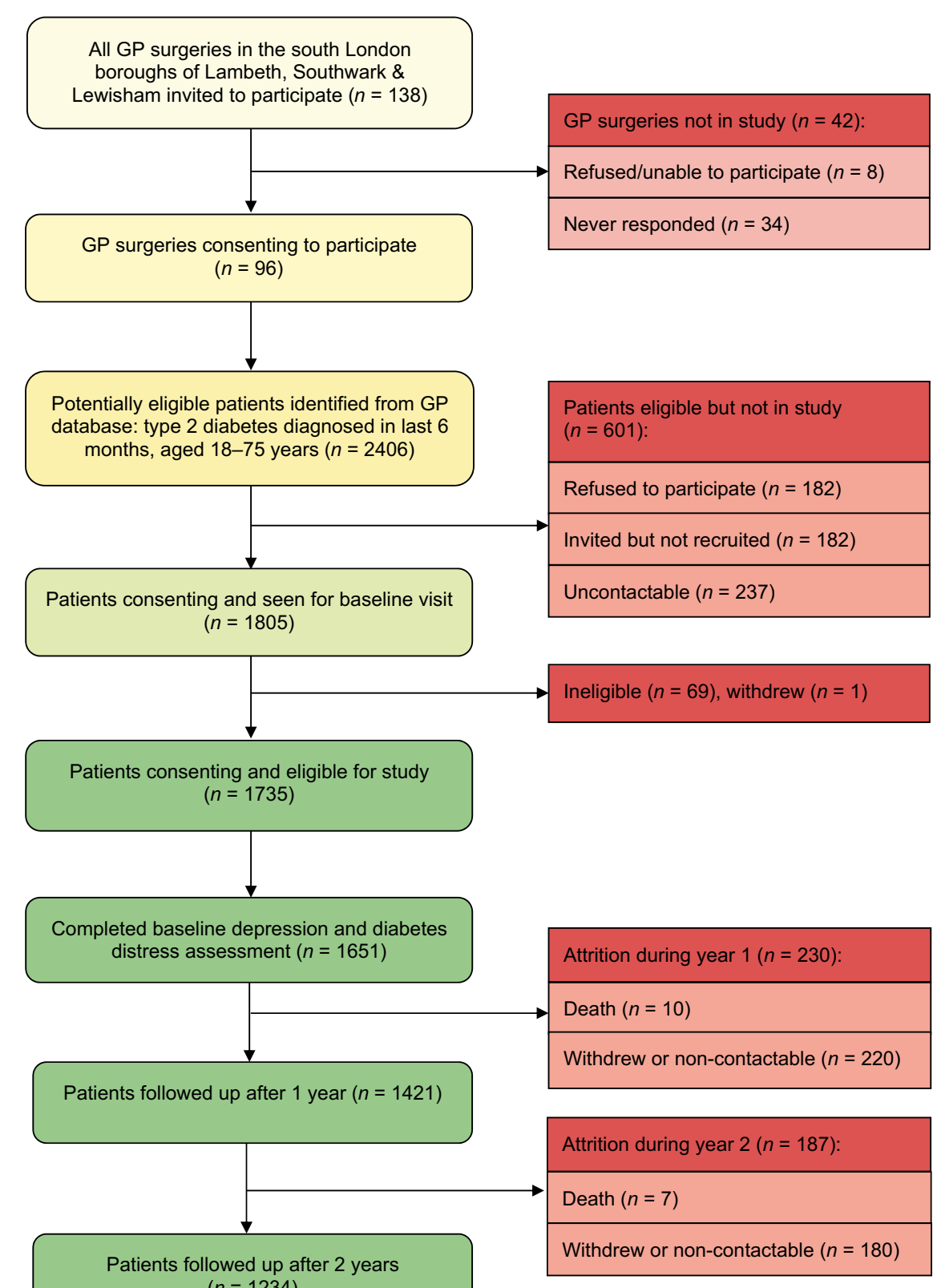

Patients followed up after 2 years $(n=1234)$ concentration (Table 3). Similar findings were observed for continuous PHQ-9 score. For diabetes distress, there was no association with incident macrovascular complications, whether measured categorically or continuously (Table 2 and Table 3). In univariate or adjusted models, there was no association between either depressive symptoms or diabetes distress (measured categorically or continuously) and incident microvascular complications (Table 2 and Table 3 ).

\section{Discussion}

The SOUL-D cohort is a multi-ethnic, population-based primary care cohort of people with newly diagnosed type 2 diabetes, designed and powered to test the effects of depressive symptoms on proxy markers of cardiovascular risk. Unexpectedly, we found that neither depressive symptoms nor diabetes distress were associated with worsening $\mathrm{HbA}_{1 \mathrm{c}}$ after 2 years. Despite a low number of overall events, depressive symptoms at diagnosis of type 2 diabetes were associated with greater risk for macrovascular complications during 2 year follow-up. This occurred independently of age, sex, ethnicity and vascular risk factors but was attenuated after adjustment for serum IL-1RA. Because all the participants were recruited within 6 months of diagnosis, confounding by duration of diagnosed diabetes was minimised. 


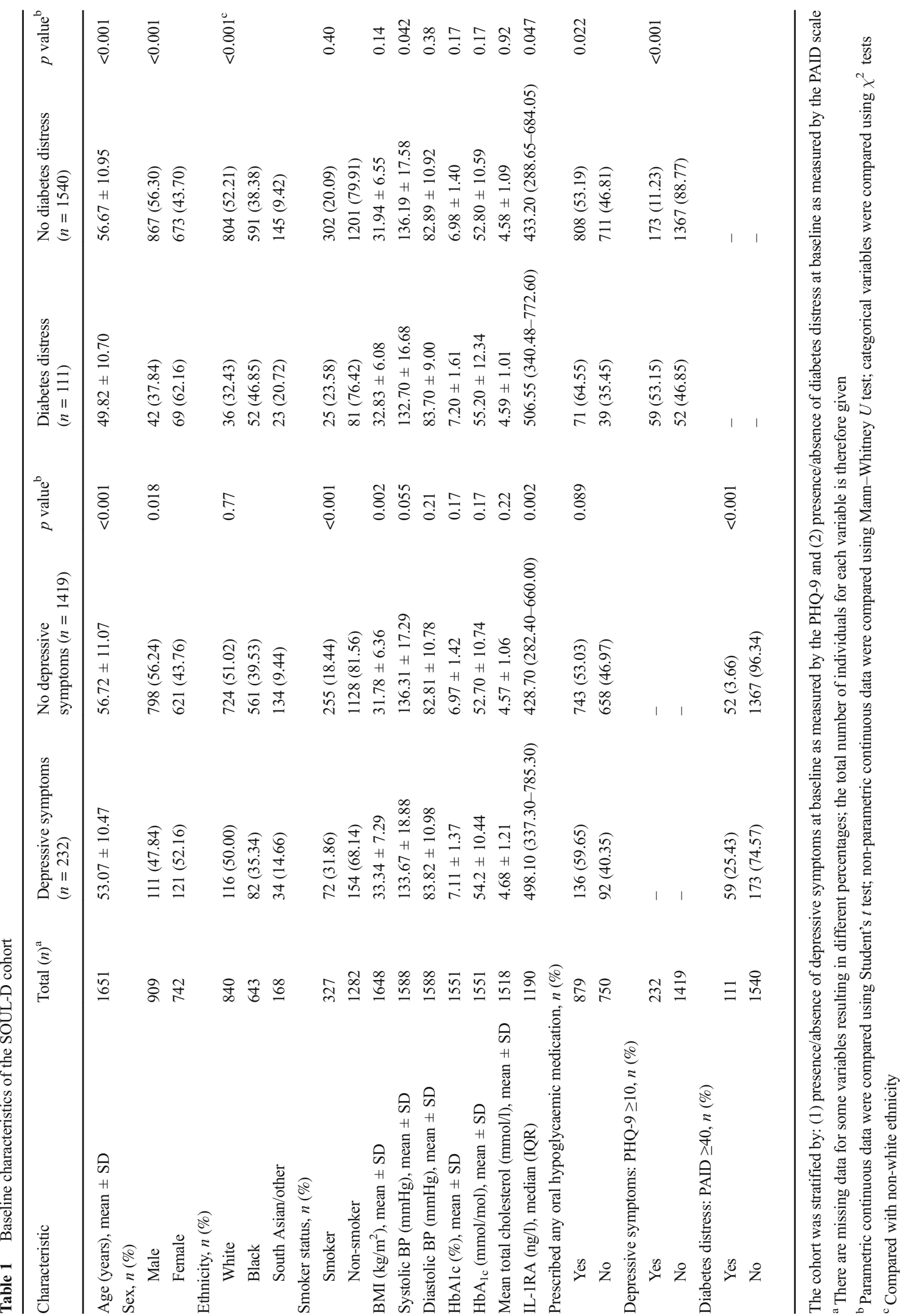


Table 2 Outcomes for the SOUL-D cohort

\begin{tabular}{|c|c|c|c|c|c|c|c|}
\hline Outcome & Total $(n)^{\mathrm{a}}$ & $\begin{array}{l}\text { Depressive } \\
\text { symptoms } \\
(n=232)\end{array}$ & $\begin{array}{l}\text { No depressive } \\
\text { symptoms } \\
(n=1419)\end{array}$ & $p$ value ${ }^{\mathrm{b}}$ & $\begin{array}{l}\text { Diabetes } \\
\text { distress } \\
(n=111)\end{array}$ & $\begin{array}{l}\text { No diabetes } \\
\text { distress } \\
(n=1540)\end{array}$ & $p$ value $^{\mathrm{b}}$ \\
\hline \multicolumn{8}{|l|}{ Psychological } \\
\hline \multicolumn{8}{|c|}{ Depressive symptoms: PHQ- $9 \geq 10$ at 2 years, $n(\%)$} \\
\hline Yes & 162 & $82(54.30)$ & $80(7.82)$ & \multirow[t]{2}{*}{$<0.001$} & $34(45.95)$ & $128(11.63)$ & \multirow[t]{2}{*}{$<0.001$} \\
\hline No & 1012 & $69(45.70)$ & $943(92.18)$ & & $40(54.05)$ & $972(88.37)$ & \\
\hline \multicolumn{8}{|c|}{ Diabetes distress: PAID $\geq 40$ at 2 years, $n(\%)$} \\
\hline Yes & 28 & $17(11.64)$ & $11(1.09)$ & \multirow[t]{2}{*}{$<0.001$} & $12(17.14)$ & $16(1.47)$ & \multirow[t]{2}{*}{$<0.001$} \\
\hline No & 1128 & $129(88.36)$ & 999 (98.91) & & $58(82.86)$ & $1070(98.53)$ & \\
\hline \multicolumn{8}{|l|}{ Glycaemic control } \\
\hline 1 year $\mathrm{HbAlc}(\%)$, mean $\pm \mathrm{SD}$ & 1421 & $6.98(1.30)$ & $6.89(1.27)$ & 0.38 & $6.79(1.34)$ & $6.91(1.27)$ & 0.69 \\
\hline 1 year $\mathrm{HbA}_{1 \mathrm{c}}(\mathrm{mmol} / \mathrm{mol})$, mean $\pm \mathrm{SD}$ & 1421 & $52.80(9.83)$ & $51.80(9.55)$ & 0.38 & $51.0(10.3)$ & $51(9.56)$ & 0.69 \\
\hline 2 year $\mathrm{HbAlc}(\%)$, mean $\pm \mathrm{SD}$ & 1234 & $7.13(1.32)$ & $6.89(1.28)$ & 0.035 & $7.14(1.43)$ & $6.91(1.28)$ & 0.16 \\
\hline 2 year $\mathrm{HbA}_{\mathrm{lc}}(\mathrm{mmol} / \mathrm{mol})$, mean $\pm \mathrm{SD}$ & 1234 & $54.40(10.07)$ & $51.80(9.62)$ & 0.035 & $54.50(10.87)$ & $52.00(9.63)$ & 0.16 \\
\hline \multicolumn{8}{|l|}{ Complications } \\
\hline Incident MI or CABG by 2 years, $n(\%)$ & & & & & & & \\
\hline Yes & 24 & $7(4.12)$ & $17(1.58)$ & \multirow[t]{2}{*}{0.031} & $2(2.50)$ & $22(1.89)$ & \multirow[t]{2}{*}{0.40} \\
\hline No & 1221 & $163(95.88)$ & $1058(98.42)$ & & $78(97.50)$ & $1143(98.91)$ & \\
\hline \multicolumn{8}{|l|}{ Incident stroke by 2 years, $n(\%)$} \\
\hline Yes & 12 & $3(1.78)$ & $9(0.84)$ & \multirow[t]{2}{*}{0.26} & $0(0.00)$ & $12(1.03)$ & \multirow[t]{2}{*}{1.0} \\
\hline No & 1230 & $166(98.22)$ & $1064(99.16)$ & & $80(100.00)$ & $1150(98.97)$ & \\
\hline \multicolumn{8}{|c|}{ Any incident carotid/limb revascularisation or amputation by 2 years, $n(\%)$} \\
\hline Yes & 13 & $2(1.18)$ & $11(1.02)$ & \multirow[t]{2}{*}{0.86} & $1(1.25)$ & $12(1.03)$ & \multirow[t]{2}{*}{0.79} \\
\hline No & 1231 & $168(98.82)$ & $1063(98.98)$ & & $79(98.75)$ & $1152(98.97)$ & \\
\hline \multicolumn{8}{|c|}{ Any incident macrovascular complication by 2 years, $n(\%)$} \\
\hline At least one & 40 & $10(5.88)$ & $30(2.79)$ & \multirow[t]{2}{*}{0.037} & $3(3.75)$ & $37(3.17)$ & \multirow[t]{2}{*}{0.50} \\
\hline None & 1207 & $160(94.12)$ & $1047(97.21)$ & & $77(96.25)$ & $1130(96.83)$ & \\
\hline \multicolumn{8}{|l|}{ Retinopathy present by 2 years, $n(\%)$} \\
\hline Yes & 162 & $27(16.67)$ & $135(13.26)$ & \multirow[t]{2}{*}{0.24} & $15(17.65)$ & $147(13.42)$ & \multirow[t]{2}{*}{0.39} \\
\hline No & 1018 & $135(83.33)$ & $883(86.74)$ & & $70(82.35)$ & $948(86.58)$ & \\
\hline Neuropathy present by 2 years, $n(\%)$ & & & & & & & \\
\hline Yes & 113 & $13(9.70)$ & $100(10.49)$ & 0.78 & $3(4.23)$ & $110(10.83)$ & 0.13 \\
\hline No & 974 & $121(90.30)$ & $853(89.51)$ & & $68(95.77)$ & $906(89.17)$ & \\
\hline Nephropathy present by 2 years (\%) & & & & & & & \\
\hline Yes & 156 & $22(16.06)$ & $134(15.40)$ & 0.90 & $12(17.65)$ & $144(15.33)$ & 0.48 \\
\hline No & 851 & $115(83.94)$ & $736(84.60)$ & & $56(82.35)$ & $795(84.67)$ & \\
\hline Any microvascular complication by 2 ye & $\mathrm{s}, n(\%)$ & & & & & & \\
\hline At least one & 388 & $55(30.73)$ & $333(28.83)$ & 0.60 & $29(31.52)$ & $359(28.90)$ & 0.54 \\
\hline None & 946 & $124(69.27)$ & $822(71.17)$ & & $63(68.48)$ & $883(71.10)$ & \\
\hline Diabetes treatment & & & & & & & \\
\hline Prescribed insulin at 2 years $(\%)$ & & & & & & & \\
\hline Yes & 54 & $14(7.65)$ & $40(3.43)$ & 0.008 & $7(7.78)$ & 47 (3.73) & 0.065 \\
\hline No & 1296 & 169 (92.35) & 1127 (96.57) & & $83(92.22)$ & $1213(96.27)$ & \\
\hline Prescribed diabetes medication at 2 year & $n(\%)$ & & & & & & \\
\hline Yes & 894 & $137(74.86)$ & $757(64.87)$ & 0.008 & $73(80.22)$ & $821(65.21)$ & 0.004 \\
\hline No & 456 & $46(25.14)$ & $410(35.13)$ & & $18(19.78)$ & $438(34.79)$ & \\
\hline
\end{tabular}

The cohort was stratified by: (1) presence/absence of depressive symptoms at baseline as measured by the PHQ-9 and (2) presence/absence of diabetes distress status at baseline as measured by the PAID scale

${ }^{a}$ There are missing data for some variables resulting in different percentages; the total number of individuals for each variable is therefore given

${ }^{\mathrm{b}}$ Parametric continuous data were compared using Student's $t$ test; non-parametric continuous data were compared using Mann-Whitney $U$ test; categorical variables were compared using $\chi^{2}$ tests

\section{Comparison with other studies}

\section{Prevalence of diabetes distress and depressive symptoms} The low prevalence of diabetes distress in the SOUL-D cohort is comparable with other primary care samples [38]. Hospital cohorts have generally reported much higher prevalence of diabetes distress $[22,38]$ but this may relate to a bias towards people with more complex and complicated diabetes in these services. Like other studies, we found that depressive symptoms and diabetes distress, although correlated, overlapped in only a minority of individuals [19].

Glycaemic control After adjustment for confounders including baseline glycaemic control, neither depressive symptoms nor diabetes distress were associated with worse glycaemic control after 2 years in our study. In two previous prospective 


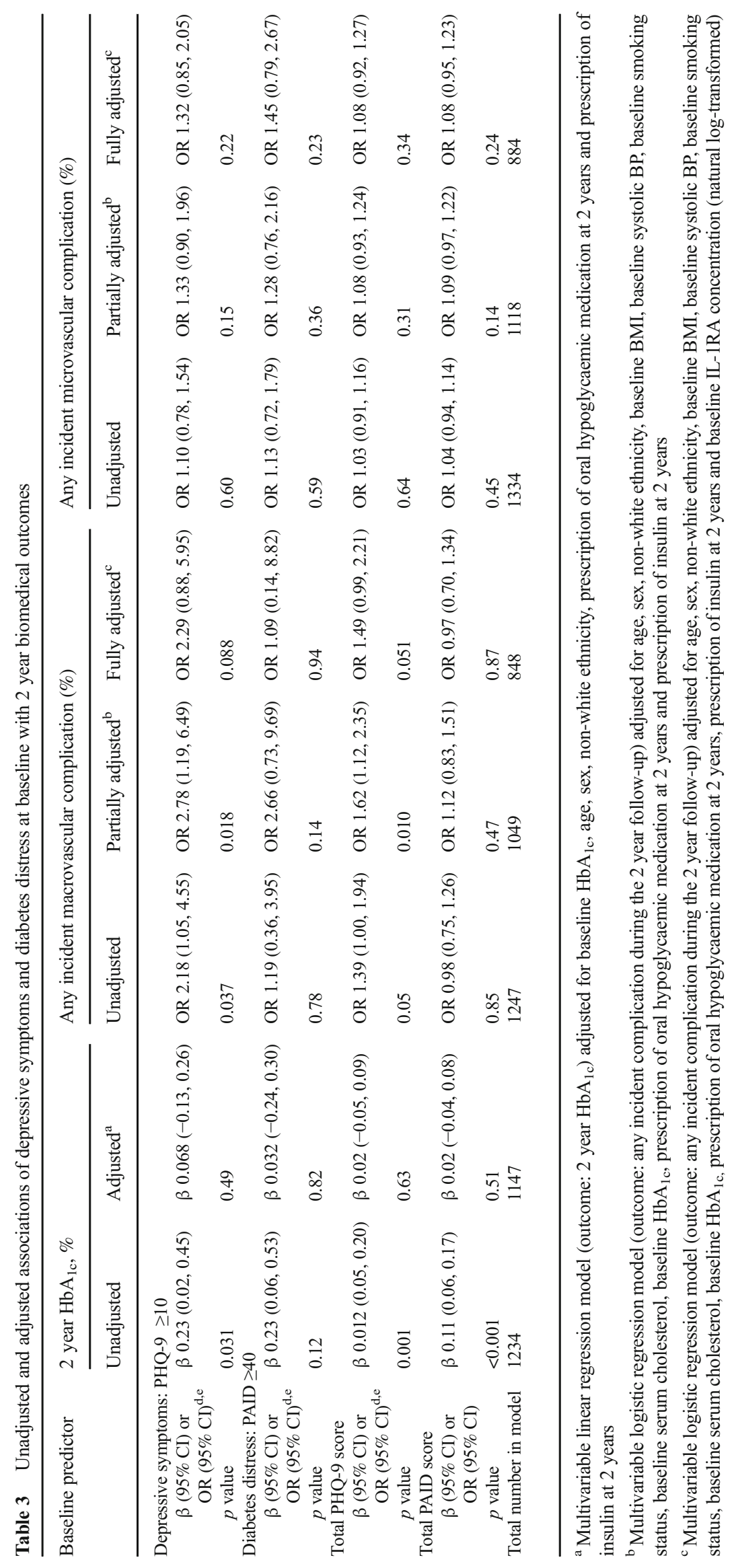


studies of depressive symptoms, but not diabetes distress, any differences in glycaemic control were likewise negated by adjusting for baseline glycaemic control [10, 11]. In comparing diabetes distress and depressive symptoms, a study of 3305 Japanese individuals with type 2 diabetes reported that diabetes distress was associated with greater odds of poor glycaemic control $\left(\mathrm{HbA}_{1 \mathrm{c}} \geq 8 \%\right)$ than depressive symptoms [26]. However, that cohort differed from ours by the longestablished diabetes of its participants (mean 13.8 years, $41 \%$ receiving insulin therapy). Likewise, a study of 627 individuals in a secondary care setting in the Netherlands reported that diabetes distress was more strongly associated with glycaemic control than depressive symptoms [22]. Both studies were limited by their cross-sectional design. Only one previous observational study in a type 2 diabetes cohort has compared depressive symptoms and diabetes distress for their prospective associations with glycaemic control [9]. In this study, Fisher and colleagues reported a significant association between diabetes distress and worsening glycaemic control, an association not demonstrated for depressive symptoms. Although comparable with ours in recruiting a multi-ethnic, primary care cohort, the Fisher study was limited by a smaller sample size $(n=506)$, shorter follow-up, longer mean duration of diabetes (8.1 years) and lack of data on incident complications.

Complications A previous meta-analysis found that depressive symptoms were associated with both macrovascular and microvascular complications in individuals with type 1 and type 2 diabetes [4]. Several studies have demonstrated that depressive symptoms are associated with incident stroke in individuals with type 2 diabetes [39, 40]. For diabetes distress, one previous prospective cohort study reported that general distress (rather than diabetes-specific distress) was associated with a 1.7 -fold increased risk of cardiovascular disease over time [41]. To date, however, we are not aware of any study that has investigated the prospective association between diabetes distress and depressive symptoms with incident complications.

\section{Interpretation}

Our findings were unexpected. If the effect of depression resulted from a behavioural mechanism such as self-neglect and poor adherence to medication, it is reasonable that the consequences would manifest as poorer glycaemic control within the first 2 years of diagnosis. We did not observe this but we did observe an effect of depression on macrovascular disease. While this may reflect pre-existing vulnerability to cardiovascular disease, it could be that depression is also a proxy marker for future cardiovascular risk. Although the number of events was low, we found that the association between depressive symptoms and incident macrovascular disease occurred independently of other known vascular risk factors and glycaemic control. When we further controlled for baseline IL-1RA concentration, the association between depressive symptoms and macrovascular disease was attenuated, pointing towards a possible inflammatory mechanism. There is compelling evidence that elevated inflammation is on the causal pathway for the pathogenesis of type 2 diabetes and subsequent macrovascular complications [42, 43]. Intervention studies that would allow testing of the directionality of the association between depressive symptoms, inflammation and biomedical outcomes are needed. Future studies should take into account wider contextual factors, such as frequency of contact with healthcare professionals, access to diabetes education and access to mental health services.

\section{Limitations}

Our study has several limitations. Despite its inner-city setting with high overall levels of deprivation, we were able to achieve nearly $70 \%$ GP participation. Although there is a risk of selection bias compared with those not participating, there was no difference in deprivation indicators between those surgeries participating and those not. There is a risk of attrition bias due to dropout at 2 years, although neither of the explanatory variables were significant predictors of attrition. The low prevalence of diabetes distress may have restricted power when testing its associations. We used a self-report measure of depressive symptoms, which is well suited to epidemiological studies but will likely result in more cases of depressive symptoms when compared with a diagnostic interview. Although our findings were supported by both categorical and continuous measures of depressive symptoms, the overall number of macrovascular events during follow-up was low. We did not control for previous cardiovascular disease as this variable may be collinear, being on the causal pathway to future cardiovascular disease, and addition of this covariate would have reduced the number of events per cell. Likewise, because of low numbers of events, effect modification analysis stratified by inflammation status was not possible and IL1RA could only be included as a confounder in the analyses. The latter two limitations will only be overcome with longerterm follow-up, by which time a greater number of macrovascular events is expected.

\section{Conclusion}

We conclude from our study that neither depressive symptoms nor diabetes distress is associated with worsening glycaemic control over 2 years. Conversely, depressive symptoms are independently associated with increased risk of incident macrovascular disease in the first 2 years of type 2 diabetes. As the overall number of events was low, longer-term prospective studies and intervention studies are now needed to test whether depressive symptoms at diagnosis of type 2 diabetes are a modifiable target. 
Acknowledgements The authors would like to thank the people who volunteered to take part in this study. The authors also thank the SOUL-D research team, King's College London, London, UK (J. Schonbeck, J. Valka, N. Iles, B. Jackson, E. Britneff, L. East, J. Hunt, S. Mann, G. Knight, L. Marwood, R. Stopford, J. P. Laake, K. Twist, M. Hussain and A. Bayley), the staff at participating general practices, the Primary Care Research Network (PCRN-GL) for their assistance in recruiting the general practices, the Diabetes Research Network and the South London Comprehensive Local Research network.

Data availability The datasets generated during and/or analysed during the current study are available from the corresponding author on reasonable request.

Funding This report presents independent research funded by the National Institute for Health Research (NIHR) under its Programme Grants for Applied Research scheme (RP-PG-0606-1142) and partfunded by the NIHR Biomedical Research Centre at South London and South London and Maudsley NHS Foundation Trust. Additional funding was received from the EFSD/Lilly Mental Health in Diabetes Programme (2010) and the NIHR Biomedical Research Centre for Mental Health at the South London and Maudsley NHS Foundation Trust and Institute of Psychiatry, Psychology and Neuroscience, King's College London. The views expressed in this publication are those of the authors and not necessarily those of the NHS, the NIHR or the Department of Health. CM receives salary support from a fellowship funded by the Royal College of Physicians of Edinburgh. The study sponsor was not involved in the design of the study; the collection, analysis, and interpretation of data; writing the report; or the decision to submit the report for publication.

Duality of interest KI has received honoraria from Eli Lilly, Sanofi, Janssen and Sunovion for lectures at educational events. KW has received an honorarium from Atlantis Healthcare. JCP has received speaker and/or consultancy fees from Medtronic, Roche, CeQur, Cellnovo, Eli Lilly and Novo Nordisk. SAA has served on advisory boards for Novo Nordisk, Medtronic and Roche. All other authors declare that there is no duality of interest associated with their contribution to this manuscript.

Contribution statement KI, SAA, KW, SMT and DS designed and created the SOUL-D cohort. KI, CM and SAA co-authored the first draft. $\mathrm{CDM}$ and DS performed the statistical analyses. KW led the data collection. RAS led the laboratory analysis. JCP provided further interpretation of data. KW, JCP, SMT, RAS and DS revised the manuscript for important intellectual content. All authors approved the final manuscript. KI is responsible for the integrity of the work as a whole.

Open Access This article is distributed under the terms of the Creative Commons Attribution 4.0 International License (http:// creativecommons.org/licenses/by/4.0/), which permits unrestricted use, distribution, and reproduction in any medium, provided you give appropriate credit to the original author(s) and the source, provide a link to the Creative Commons license, and indicate if changes were made.

\section{References}

1. Mezuk B, Eaton WW, Albrecht S, Golden SH (2008) Depression and type 2 diabetes over the lifespan: a meta-analysis. Diabetes Care 31:2383-2390

2. Nouwen A, Winkley K, Twisk J et al (2010) Type 2 diabetes mellitus as a risk factor for the onset of depression: a systematic review and meta-analysis. Diabetologia 53:2480-2486
3. Anderson RJ, Freedland KE, Clouse RE, Lustman PJ (2001) The prevalence of comorbid depressive symptoms in adults with diabetes: a meta-analysis. Diabetes Care 24:1069-1078

4. de Groot M, Anderson R, Freedland KE, Clouse RE, Lustman PJ (2001) Association of depressive symptoms and diabetes complications: a meta-analysis. Psychosom Med 63:619-630

5. Winkley K, Sallis H, Kariyawasam D et al (2012) Five-year followup of a cohort of people with their first diabetic foot ulcer: the persistent effect of depressive symptoms on mortality. Diabetologia 55:303-310

6. Katon WJ, Lin EH, Russo J et al (2004) Cardiac risk factors in patients with diabetes mellitus and major depressive symptoms. J Gen Intern Med 19:1192-1199

7. Katon WJ, Russo JE, Heckbert SR et al (2010) The relationship between changes in depressive symptoms symptoms and changes in health risk behaviors in patients with diabetes. Int $\mathrm{J}$ Geriatr Psychiatry 25:466-475

8. Lustman PJ, Anderson RJ, Freedland KE, de Groot M, Carney RM, Clouse RE (2000) Depression and poor glycaemic control: a metaanalytic review of the literature. Diabetes Care 23:934-942

9. Fisher L, Mullan JT, Arean P, Glasgow RE, Hessler D, Masharani U (2010) Diabetes distress but not clinical depressive symptoms or depressive symptoms is associated with glycaemic control in both cross-sectional and longitudinal analyses. Diabetes Care 33:23-28

10. Palta P, Golden SH, Teresi JA et al (2014) Depression is not associated with diabetes control in minority elderly. J Diabetes Complicat 28:798-804

11. Aikens JE, Perkins DW, Lipton B, Piette JD (2009) Longitudinal analysis of depressive symptoms and glycaemic control in type 2 diabetes. Diabetes Care 32:1177-1181

12. Chiu CJ, Wray LA, Beverly EA, Dominic OG (2010) The role of health behaviors in mediating the relationship between depressive symptoms and glycaemic control in type 2 diabetes: a structural equation modeling approach. Soc Psychiatry Psychiatr Epidemiol 45:67-76

13. Dirmaier J, Watzke B, Koch U et al (2010) Diabetes in primary care: prospective associations between depressive symptoms, nonadherence and glycaemic control. Psychother Psychosom 79: 172-178

14. Lustman PJ, Clouse RE, Nix BD et al (2006) Sertraline for prevention of depressive symptoms recurrence in diabetes mellitus: a randomized, double-blind, placebo-controlled trial. Arch Gen Psychiatry 63:521-529

15. Lustman PJ, Griffith LS, Freedland KE, Kissel SS, Clouse RE (1998) Cognitive behavior therapy for depressive symptoms in type 2 diabetes mellitus. A randomized, controlled trial. Ann Intern Med 129:613-621

16. Katon WJ, Lin EH, Von Korff M et al (2010) Collaborative care for patients with depressive symptoms and chronic illnesses. N Engl J Med 363:2611-2620

17. Polonsky WH, Anderson BJ, Lohrer PA et al (1995) Assessment of diabetes-related diabetes distress. Diabetes Care 18:754-760

18. Snoek FJ, Bremmer MA, Hermanns N (2015) Constructs of depressive symptoms and diabetes distress in diabetes: time for an appraisal. Lancet Diabetes Endocrinol 3:450-460

19. Snoek FJ, Kersch NY, Eldrup E et al (2012) Monitoring of Individual Needs in Diabetes (MIND)-2: follow-up data from the cross-national Diabetes Attitudes, Wishes, and Needs (DAWN) MIND study. Diabetes Care 35:2128-2132

20. Schmitt A, Reimer A, Kulzer B, Haak T, Gahr A, Hermanns N (2014) Assessment of diabetes acceptance can help identify patients with ineffective diabetes self-care and poor diabetes control. Diabet Med 31:1446-1451

21. Schmitt A, Reimer A, Kulzer B, Haak T, Gahr A, Hermanns N (2014) Negative association between depressive symptoms and 
diabetes control only when accompanied by diabetes-specific distress. J Behav Med 38:556-564

22. van Bastelaar KM, Pouwer F, Geelhoed-Duijvestijn PH et al (2010) Diabetes-specific emotional diabetes distress mediates the association between depressive symptoms and glycaemic control in type 1 and type 2 diabetes. Diabet Med 27:798-803

23. Hermanns N, Schmitt A, Gahr A et al (2015) The effect of a Diabetes-Specific Cognitive Behavioral Treatment Program (DIAMOS) for patients with diabetes and subclinical depressive symptoms: results of a randomized controlled trial. Diabetes Care 38:551-560

24. Bruce DG, Davis WA, Starkstein SE, Davis TM (2005) A prospective study of depressive symptoms and mortality in patients with type 2 diabetes: the Fremantle Diabetes Study. Diabetologia 48: 2532-2539

25. Kasteleyn MJ, de Vries L, van Puffelen AL et al (2015) Diabetesrelated diabetes distress over the course of illness: results from the Diacourse study. Diabet Med 32:1617-1624

26. Tsujii S, Hayashino Y, Ishii H, the Diabetes Distress and Care Registry at Tenri Study Group (2012) Diabetes distress, but not depressive symptoms, is associated with glycaemic control among Japanese patients with type 2 diabetes: Diabetes Distress and Care Registry at Tenri (DDCRT 1). Diabet Med 29:1451-1455

27. Winkley K, Thomas SM, Sivaprasad S et al (2013) The clinical characteristics at diagnosis of type 2 diabetes in a multi-ethnic population: the South London Diabetes cohort (SOUL-D). Diabetologia 56:1272-1281

28. Alberti KG, Zimmet PZ (1998) Definition, diagnosis and classification of diabetes mellitus and its complications. Part 1: diagnosis and classification of diabetes mellitus provisional report of a WHO consultation. Diabet Med 15:539-553

29. Kroenke K, Spitzer RL, Williams JB (2001) The PHQ-9: validity of a brief depressive symptoms severity measure. J Gen Intern Med $16: 606-613$

30. Twist K, Stahl D, Amiel SA, Thomas S, Winkley K, Ismail K (2013) Comparison of depressive symptoms in type 2 diabetes using a two-stage survey design. Psychosom Med 75:791-797

31. Welch GW, Jacobson AM, Polonsky WH (1997) The problem areas in diabetes scale. An evaluation of its clinical utility. Diabetes Care 20:760-766

32. Snoek FJ, Pouwer F, Welch GW, Polonsky WH (2000) Diabetesrelated emotional distress in Dutch and U.S. diabetic patients: cross- cultural validity of the problem areas in diabetes scale. Diabetes Care 23:1305-1309

33. Fenwick EK, Rees G, Holmes-Truscott E, Browne JL, Pouwer F, Speight $J$ (2016) What is the best measure for assessing diabetes distress? A comparison of the Problem Areas in Diabetes and Diabetes Distress Scale: results from Diabetes MILES-Australia. J Health Psychol doi:10.1177/1359105316642006

34. Raymond MR (1986) Missing data in evaluation research. Eval Health Prof 9:395-420

35. Roth PL, Switzer FS, Switzer DM (1999) Missing data in multiple item scales: a Monte Carlo analysis of missing data. Organ Res Methods 2:211-232

36. Department for Communities and Local Government (2010) English indices of deprivation 2010. Available from www.gov.uk/ government/statistics/english-indices-of-deprivation-2010 (archived). Accessed 15 May 2017

37. Laake JP, Stahl D, Amiel SA et al (2014) The association between depressive symptoms and systemic inflammation in people with type 2 diabetes: findings from the South London Diabetes Study. Diabetes Care 37:2186-2192

38. Stoop CH, Nefs G, Pop VJ et al (2014) Diabetes-specific emotional diabetes distress in people with type 2 diabetes: a comparison between primary and secondary care. Diabet Med 31:1252-1259

39. Ting RZ, Lau ES, Ozaki R, et al. High risk for cardiovascular disease in Chinese type 2 diabetic patients with major depressive symptoms - a 7-year prospective analysis of the Hong Kong Diabetes Registry. J Affect Disord 149:129-135

40. Cummings DM, Kirian K, Howard G et al (2016) Consequences of comorbidity of elevated stress and/or depressive symptoms and incident cardiovascular outcomes in diabetes: results from the REasons for Geographic And Racial Differences in Stroke (REGARDS) study. Diabetes Care 39:101-109

41. Dalsgaard EM, Vestergaard M, Skriver MV et al (2014) Psychological diabetes distress, cardiovascular complications and mortality among people with screen-detected type 2 diabetes: follow-up of the ADDITION-Denmark trial. Diabetologia 57: 710-717

42. Pickup JC, Mattock MB (2003) Activation of the innate immune system as a predictor of cardiovascular mortality in type 2 diabetes mellitus. Diabet Med 20:723-726

43. Herder C, Dalmas E, Böni-Schnetzler M, Donath MY (2015) The IL-1 pathway in type 2 diabetes and cardiovascular complications. Trends Endocrinol Metab 26:551-563 